\title{
PLANAR POSETS THAT ARE ACCESSIBLE FROM BELOW HAVE DIMENSION AT MOST 6
}

\author{
CSABA BIRÓ, BARTŁOMIEJ BOSEK, HEATHER C. SMITH, WILLIAM T. TROTTER, \\ RUIDONG WANG, AND STEPHEN J. YOUNG
}

\begin{abstract}
Planar posets can have arbitrarily large dimension. However, a planar poset of height $h$ has dimension at most $192 h+96$, while a planar poset with $t$ minimal elements has dimension at most $2 t+1$. In particular, a planar poset with a unique minimal element has dimension at most 3 . In this paper, we extend this result by showing that a planar poset has dimension at most 6 if it has a plane diagram in which every minimal element is accessible from below.
\end{abstract}

\section{INTRODUCTION}

A non-empty family $\mathcal{R}$ of linear extensions of a poset $P$ is called a realizer of $P$ when $x \leq y$ in $P$ if and only if $x \leq y$ in $L$ for each $L \in \mathcal{R}$. The dimension of a poset $P$, as defined by Dushnik and Miller in their seminal paper [3, is the least positive integer $d$ for which $P$ has a realizer $\mathcal{R}$ with $|\mathcal{R}|=d$.

In recent years, there has been considerable interest in bounding the dimension of a poset in terms of graph theoretic properties of its cover graph and its order diagram. For example, the following papers link the dimension of a poset with tree-width, forbidden minors, sparsity and game coloring numbers: 6], [12, 20, [7] and [8]. The results presented here continue in this theme.

Recall that a poset $P$ is said to be planar if its order diagram (also called a Hasse diagram) can be drawn without edge crossings in the plane. As is well known, a planar poset has an order diagram without edge crossings in which edges are straight line segments. Nevertheless, we elect to consider order diagrams in which covering edges can be piecewise linear, as this convention simplifies our illustrations. Given a planar poset $P$, a drawing of the order diagram of $P$ using piecewise linear paths for edges such that there are no edge crossings will simply be called a plane diagram of $P$.

In discussing a plane diagram $\mathbb{D}$ for a poset $P$, we will assume, without loss of generality, that no two points of $P$ lies on the same horizontal or vertical line in the plane. We will also discuss points in the plane which do not correspond to elements of $P$. In particular, the set of points in the plane which do not correspond to elements of $P$ and do not lie on the piecewise linear covering edges in $\mathbb{D}$ is partitioned into one or more simply connected regions. In general, there can be arbitrarily many bounded regions, however the boundaries of these regions need not

Date: June 19, 2019.

2010 Mathematics Subject Classification. 06A07, 05C35.

Key words and phrases. Dimension, planar poset, accessible from below poset.

PNNL Information Release: PNNL-SA-144431.

B. Bosek is supported by Polish National Science Center grant 2013/11/D/ST6/03100. 

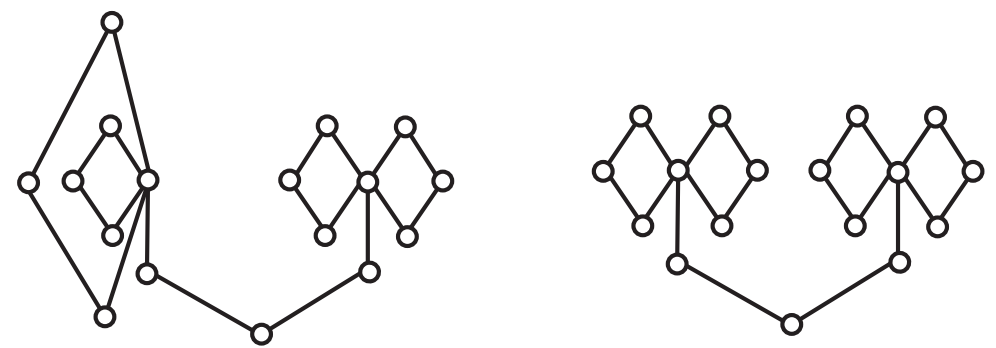

Figure 1. The diagram on the right is an AFB-Diagram

be simple closed curves. Among these regions, there is always a unique, unbounded region which is usually referred to as the exterior region.

Let $\mathbb{D}$ be a plane diagram for poset $P$, and let $x$ be a minimal element of $P$. We will say that $x$ is accessible from below when there is a positive number $\epsilon=\epsilon(x)$ so that any point $p$ in the plane which is distinct from $x$, on the vertical ray emanating downwards from $x$ and within distance $\epsilon$ from $x$ is in the exterior region. In turn we say that a plane diagram $\mathbb{D}$ is accessible from below if if every minimal element of $P$ is accessible from below.

We find it convenient to abbreviate the phrase "accessible from below" with the acronym AFB, so we will say that a minimal element $x$ is AFB in a diagram $\mathbb{D}$, and we will refer to an AFB-diagram. We then say that a poset $P$ is an AFB-poset if it has an AFB-diagram. All AFB-posets are planar, but there are planar posets which are not AFB. Also, an AFB-poset can have many plane diagrams with some of them AFB-diagrams and others not. We illustrate this situation in Figure 1 where we show two plane diagrams of an AFB-poset $P$. The diagram on the right is an AFB-diagram while the diagram on the left is not.

The principal goal of this paper is to prove the following upper bound on the dimension of an AFB-poset.

Theorem 1. If $P$ is an AFB-poset, then $\operatorname{dim}(P) \leq 6$.

The remainder of this paper is organized as follows. Some background material necessary for the proof of Theorem 1 is summarized in the next section, and the proof our main theorem is given in Section 3 . We close in Section 4 with some comments on the motivation for this line of research and connections with open problems.

We note that for every $d \geq 6$, it is an easy exercise to construct an AFB-poset $P$ for which one cannot argue that $\operatorname{dim}(P) \leq d$ by any of the other known results for planar posets. Although we do not know if our upper bound is best possible, as detailed in Section 4, a finite upper bound on the dimension of AFB-posets is sufficient for our long range goals.

\section{BACKGROUND MATERIAL}

We use (essentially) the same notation and terminology for working with dimension as has been employed by several authors in recent papers, including: 5], 14, [6, [18] and [19], so our treatment will be concise.

Let $P$ be a poset with linear extension $L$ and let $(x, y) \in \operatorname{Inc}(P)$. We say $L$ reverses $(x, y)$ when $x>y$ in $L$. When $S \subset \operatorname{Inc}(P)$, we say that $L$ reverses $S$ when 
$L$ reverses every pair in $S$. When $\mathcal{R}$ is a family of linear extensions of $P$, we say $\mathcal{R}$ reverses $S$ when, for each $(x, y) \in S$, there is some $L \in \mathcal{R}$ such that $L$ reverses $(x, y)$. Evidently, the dimension of $P$ is just the minimum size of a non-empty family of linear extensions which reverses $\operatorname{Inc}(P)$.

In the discussion to follow, we sometimes express a linear order on a finite set by writing $\left[u_{1}<u_{2}<\cdots<u_{r}\right]$, for example.

A subset $S \subset \operatorname{Inc}(P)$ is reversible when there is a linear extension $L$ of $P$ which reverses $S$. When $k \geq 2$, a sequence $\left\{\left(x_{i}, y_{i}\right): 1 \leq i \leq k\right\}$ of incomparable pairs in $P$ is called an alternating cycle (of length $k$ ) when $x_{i} \leq y_{i+1}$ in $P$ for all $i \in[k]$, which should be interpreted cyclically, i.e., we also intend that $x_{k} \leq y_{1}$ in $P$. For the balance of the paper, we will use similar cyclic notation without further comment.

An alternating cycle is strict if for each $i \in[k], x_{i} \leq y_{j}$ in $P$ if and only if $j=i+1$, and further, the sets $\left\{x_{1}, x_{2}, \ldots, x_{k}\right\}$ and $\left\{y_{1}, y_{2}, \ldots, y_{k}\right\}$ are $k$-element antichains.

A poset has dimension 1 if and only if it is a chain. When $P$ is not a chain, the dimension of $P$ is just the least positive integer $d \geq 2$ for which there is a covering $\operatorname{Inc}(P)=S_{1} \cup S_{2} \cup \cdots \cup S_{d}$ with $S_{i}$ reversible for each $i \in[d]$, The following elementary lemma of Trotter and Moore [16] characterizing reversible sets has become an important tool in dimension theory.

Lemma 2. Let $P$ be a poset and let $S \subseteq \operatorname{Inc}(P)$. Then the following statements are equivalent.

(1) $S$ is reversible.

(2) There is no $k \geq 2$ for which $S$ contains an alternating cycle of length $k$.

(3) There is no $k \geq 2$ for which $S$ contains a strict alternating cycle of length $k$.

When $x<y$ in a poset $P$, we refer to a sequence $W[x, y]=\left(u_{1}, u_{2}, \ldots, u_{r}\right)$ of elements of $P$ as a witnessing path from $x$ to $y$ when $u_{1}=x, u_{r}=y$ and $u_{i}$ is covered by $u_{i+1}$ in $P$ whenever $1 \leq i<r$. In general, there are many different witnessing paths from $x$ to $y$ and, in most instances, it will not matter which one is chosen.

When $\mathbb{D}$ is a plane diagram for a poset $P$ and $x<y$ in $P$, we will take advantage of the fact that there is a uniquely determined "left-most" witnessing path from $x$ to $y$. Analogously, there is a uniquely determined "right-most" witnessing path from $x$ to $y$. In Figure 2, we show a portion of a plane diagram where there are a total of 12 witnessing paths from $x$ to $y$. The left-most path is shown using dotted edges while the right-most path is shown using bold face edges.

We can view a witnessing path $W[x, y]$ as a finite sequence of points of the poset $P$, but we can also view it as the simply connected (and therefore infinite) set of points in the plane which belong to the covering edges in the path. From the context of the discussion, it should be clear whether we intend a witnessing path to be simply a finite set of points from $P$ or an infinite set of points in the plane. In the same spirit, we will splice witnessing paths together to form simple closed curves in the plane. These will always be infinite sets of points.

At a critical stage in our proof, we will discuss a simple closed curve $\mathcal{E}$ such that the minimal elements of $P$ are on $\mathcal{E}$, while all other elements of $P$ are in the interior of $\mathcal{E}$.

2.1. Planar Posets with a Zero. When a poset has a unique minimal element, that element is usually referred to as a "zero." Dually, if a poset has a unique 


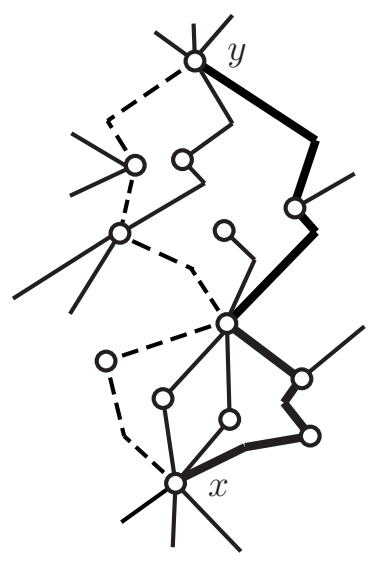

FiguRE 2. Left-most and right-most witnessing paths

maximal element, then it is called a "one." We state formally the theorem of Trotter and Moore [16, and give a short synopsis of a more modern proof given in [18, as these details will be important in proving our main theorem.

Theorem 3. If $P$ is a planar poset and $P$ has a zero, then $\operatorname{dim}(P) \leq 3$.

Since a poset and its dual have the same dimension, we also know that a planar poset with a one has dimension at most 3 .

Given a plane diagram $\mathbb{D}$ for a poset $P$ with a zero, let $L_{1}$ be the linear extension of $P$ obtrained from a depth-first search using a local left-to-right preference rule. Similarly, let $L_{2}$ be another linear extension of $P$ which is also obtained via a depth-first search, but with a right-to-left preference. As noted in [18], for every $(x, y) \in \operatorname{Inc}(P)$, exactly one of the following four statements applies:

(1) $x$ is right of $y$ (i.e. $x>y$ in $L_{1}$ and $x<y$ in $L_{2}$ ).

(2) $x$ is left of $y$ (i.e. $x<y$ in $L_{1}$ and $x>y$ in $L_{2}$ ).

(3) $x$ is outside $y$ (i.e. $x<y$ in both $L_{1}$ and $L_{2}$ ).

(4) $x$ is inside $y$ (i.e. $x>y$ in both $L_{1}$ and $L_{2}$ ).

In Figure 3 , we show a plane diagram $\mathbb{D}$ for a poset $P$ with a zero in which 10 is right of 5,7 is left of 9,14 is outside 6 and 10 is inside 13 .

Accordingly, it is natural to partition $\operatorname{Inc}(P)$ as $\mathcal{R} \cup \mathcal{L} \cup \mathcal{O} \cup \mathcal{I}$, where $\mathcal{R}$ consists of all pairs $(x, y)$ with $x$ right of $y$, etc. The binary relations $\mathcal{R}$ and $\mathcal{L}$ are complementary in the sense that $x$ is left of $y$ if and only if $y$ is right of $x$. Similarly, the binary relations $\mathcal{I}$ and $\mathcal{O}$ are complementary. Also both $\mathcal{L}$ and $\mathcal{R}$ are transitive, e.g., if $x$ is left of $y$ and $y$ is left of $z$, then $x$ is left of $z$.

The inequality $\operatorname{dim}(P) \leq 3$ is proved by showing that the following sets are reversible: (1) $\mathcal{R} \cup \mathcal{O},(2) \mathcal{L} \cup \mathcal{O}$, and (3) $\mathcal{I}$. The arguments given in [18] for the first two of these statements are constructive, as the desired linear extensions are obtained via depth-first searches. Note that the labeling used in Figure 3 results from a depth-first search using a local left-to-right preference rule. This linear extension illustrates that $\mathcal{R} \cup \mathcal{O}$ is reversible. A depth-first search using a local right-to-left preference rule will produce a linear extension reversing all pairs in $\mathcal{L} \cup \mathcal{O}$. 


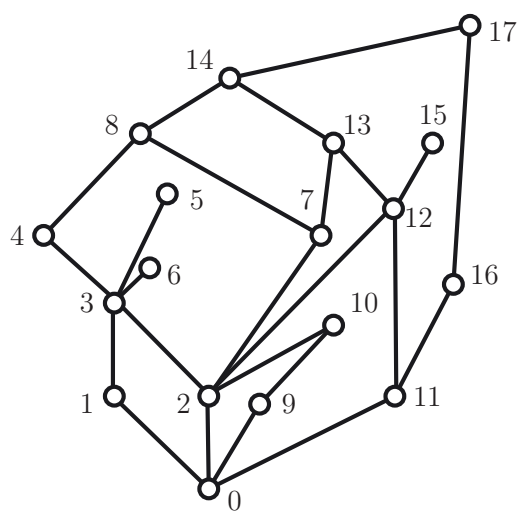

Figure 3. A planar poset with a zero

To complete the proof, it is then only necessary to show that $\mathcal{I}$ is reversible. However, as pointed out in 18, a somewhat more general result holds: planar posets with $t$ minimal elements have dimension at most $2 t+1$.

Let $\mathbb{D}$ be a plane diagram for a planar poset $P$ (with no restriction on the number of minimal elements of $P$ ). When $z \in P$, we let $U_{P}[z]$ consist of all elements $x \in P$ with $z \leq x$ in $P$. The subposet $U_{P}[z]$ is planar and $z$ is a zero. Accordingly, we can classify the incomparable pairs in $U_{P}[z]$ using the same four labels $\mathcal{R}, \mathcal{L}, \mathcal{O}$, and $\mathcal{I}$. We will say that an incomparable pair $(x, y)$ in $P$ is an enclosed pair when there is some $z \in P$ such that $x$ is inside $y$ in $U_{P}[z]$.

For the benefit of readers who may be new to arguments using alternating cycles, we give a proof for the following elementary lemma.

Lemma 4. Let $\mathbb{D}$ be a plane diagram for a poset $P$. Then the set $\mathcal{S}$ of all enclosed pairs in $P$ is reversible.

Proof. We argue by contradiction, supposing that $\mathcal{S}$ is not reversible. Then by Lemma 2, there is an integer $k \geq 2$ and a strict alternating cycle $\left\{\left(x_{i}, y_{i}\right): 1 \leq i \leq\right.$ $k\}$ of enclosed pairs. For each $i \in[k]$, let $z_{i}$ be the unique element of $P$ which is highest in the plane with $x_{i}$ inside $y_{i}$ in $U_{P}\left[z_{i}\right]$. Then let $y_{i}^{\prime}$ be the unique element of $P$ which is lowest in the plane and satisfies both $y_{i}^{\prime} \leq y_{i}$ in $P$ and $x_{i}$ is inside $y_{i}^{\prime}$ in $U_{P}\left[z_{i}\right]$. Then there are two witnessing paths $W_{1}\left[z_{i}, y_{i}^{\prime}\right]$ and $W_{2}\left[z_{i}, y_{i}^{\prime}\right]$ which form a simple closed curve $\mathcal{C}_{i}$ with $x_{i}$ in its interior.

Since $x_{i} \leq y_{i+1}$ in $P$ and $x_{i} \| y_{i}$ in $P$, it follows that $y_{i+1}$ is also in the interior of $\mathcal{C}_{i}$. Therefore $y_{i+1}$ is lower in the plane than $y_{i}^{\prime}$. This is a contradiction since this statement cannot hold for all $i \in[k]$.

In a dual manner, when $\mathbb{D}$ is a plane diagram for a poset $P$ and $z \in P$, we define $D_{P}[z]$ as the subposet consisting of all $x \in P$ with $x \leq z$ in $P$. The subposet $D_{P}[z]$ is planar, and the element $z$ is a one. Now we can classify the incomparable pairs in the subposet $D_{P}[z]$ using the same four labels but applied with the obvious dual interpretation. In general, if $(x, y)$ is an incomparable pair in a subposet of the form $D_{P}[z]$, then any of the four labels may be correct for the pair $(x, y)$. However, if $\mathbb{D}$ is an AFB-diagram for a poset $P$, then two of the four labels cannot be applicable. 
We state formally the following nearly self-evident proposition for emphasis. It does not hold for planar posets in general.

Proposition 5. Let $\mathbb{D}$ be an AFB-diagram for a poset $P$, and let $z \in P$. If $(x, y)$ is an incomparable pair in $D_{P}[z]$, then either $x$ is left of $y$ in $D_{P}[z]$ or $x$ is right of $y$ in $D_{P}[z]$. Furthermore, if $z^{\prime} \in P$ and $x, y \in D_{P}\left[z^{\prime}\right]$, then $x$ is left of $y$ in $D_{P}[z]$ if and only if $x$ is left of $y$ in $D_{P}\left[z^{\prime}\right]$.

\section{Proof of our Main Theorem}

In this section, we prove Theorem 1 i.e., we show that if $P$ is an AFB-poset, then $\operatorname{dim}(P) \leq 6$. Our first step is to reduce the problem to a somewhat simpler one.

Reduction. To show that the dimension of any AFB-poset is at most $d$, it suffices to show that whenever $\mathbb{D}$ is an AFB-diagram for a poset $P$, the set of all incomparable pairs in $\operatorname{Min}(P) \times P$ can be covered by $d-1$ reversible sets.

Proof. Let $P$ be an AFB-poset. To show that $\operatorname{dim}(P) \leq d$, we need to show that there is a covering of the set of all incomparable pairs of $P$ by $d$ reversible sets. Let $\mathbb{D}$ be an AFB-diagram for $P$. We will now show that $\mathbb{D}$ can be modified into an AFB-diagram $\mathbb{D}^{\prime}$ for a poset $P^{\prime}$ such that:

(1) $P^{\prime}$ contains $P$ as a subposet.

(2) If $(x, y)$ is an enclosed pair in $P$, then $(x, y)$ is an enclosed pair in $P^{\prime}$.

(3) If $(x, y)$ is an incomparable pair of $P$ and is not an enclosed pair in $P$, then there is a minimal element $x^{\prime} \in P^{\prime}$ with $x^{\prime} \leq x$ in $P^{\prime}$ and $x^{\prime} \| y$ in $P^{\prime}$.

We will then let $S_{0}$ consist of all incomparable pairs $(x, y)$ in $P$ such that $(x, y)$ is an enclosed pair in $P^{\prime}$. The set $S_{0}$ is reversible by Lemma 4, and it contains all enclosed pairs in $P$. It remains to consider the incomparable pairs in $P$ which are not enclosed in $P^{\prime}$. In view of the third condition for $P^{\prime}$, if the incomparable pairs $\left(x^{\prime}, y\right) \in \operatorname{Min}\left(P^{\prime}\right) \times P^{\prime}$ in $P^{\prime}$ can be covered by $d-1$ reversible sets, it follows that the set of all incomparable pairs of $P$ can be covered by $d$ reversible sets. So it only remains to explain how the poset $P^{\prime}$ should be constructed from $P$.

Let $S$ be the set of all incomparable pairs of $P$ which are not enclosed pairs in $P$ and do not belong to $\operatorname{Min}(P) \times P$. If $S=\emptyset$, simply take $\mathbb{D}^{\prime}=\mathbb{D}$ and $P^{\prime}=P$. So we may assume that $S \neq \emptyset$. Let $r=|S|$ and let $S=\left\{\left(x_{i}, y_{i}\right): 1 \leq i \leq r\right\}$ be an arbitrary labeling of the pairs in $S$.

To initialize a recursive construction, we set $\mathbb{D}_{0}=\mathbb{D}$ and $P_{0}=P$. We will now explain how to construct a sequence $\left\{\left(\mathbb{D}_{i}, P_{i}\right): 1 \leq i \leq r\right\}$ such that for each $i \in[r]$, $\mathbb{D}_{i}$ is an AFB-diagram for the poset $P_{i}$ where $M_{i}=\operatorname{Min}(P)$. The construction will ensure that $P_{i-1}$ is a subposet of $P_{i}$ and $M_{i-1}$ is a subset of $M_{i}$ whenever $1 \leq i \leq r$. Furthermore, for each $1 \leq j \leq i \leq r$, either $\left(x_{j}, y_{j}\right)$ is an enclosed pair in $P_{i}$ or there is a minimal element $x_{j}^{\prime} \in M_{j}$ such that $x_{j}^{\prime} \leq x_{j}$ in $P_{i}$ and $x_{j}^{\prime} \| y_{j}$ in $P_{i}$. The AFB-poset $P^{\prime}$ is just $P_{r}$.

Now suppose that $0 \leq i<r$ and that we have defined the AFB-diagram $\mathbb{D}_{i}$ for $P_{i}$. We then consider the pair $(x, y)=\left(x_{i+1}, y_{i+1}\right)$. Let $x^{\prime}$ be the uniquely determined element of $P$ which is lowest point in the plane and satisfies $x^{\prime} \leq x$ in $P_{i}$ and $x^{\prime} \| y$ in $P_{i}$. If $\left(x^{\prime}, y\right)$ is an enclosed pair in $P_{i}$, so is $(x, y)$. Accordingly, if $x^{\prime} \in \operatorname{Min}\left(P_{i}\right)$, or $\left(x^{\prime}, y\right)$ is an enclosed pair, we simply take $\mathbb{D}_{i+1}=\mathbb{D}_{i}$ and $P_{i+1}=P_{i}$.

Now suppose $\left(x^{\prime}, y\right)$ is not an enclosed pair in $P_{i}$, and $x^{\prime}$ is not a minimal element in $P_{i}$. It follows that $x^{\prime}$ covers one or more elements in $P_{i}$. We claim that $x^{\prime}$ has a 

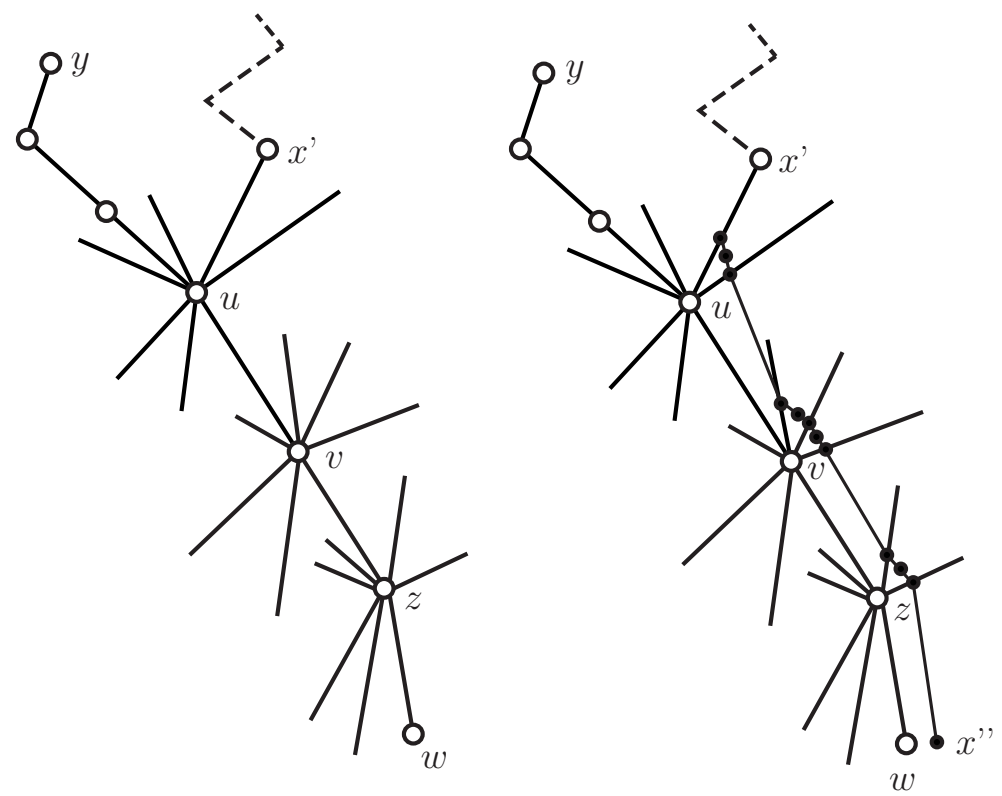

Figure 4. The Construction for the Reduction

unique lower cover. Suppose to the contrary that $x^{\prime}$ covers distinct elements $u$ and $u^{\prime}$ in $P_{i}$. In view of our choice of $x^{\prime}$, we know $u, u^{\prime} \in D_{P}[y]$. However, since $\left(x^{\prime}, y\right)$ is not an enclosed pair in $P_{i}$ and $u \| u^{\prime}$, the induced AFB-diagram of the subposet of $P_{i}$ determined by $\left\{u, u^{\prime}, x^{\prime}, y\right\}$ violates Proposition 5. The contradiction shows that $x^{\prime}$ covers a unique point $u$ as claimed.

Our choice of $x^{\prime}$ implies that $u<y$ in $P_{i}$. We consider the first edge of a witnessing path $W[u, y]$ and the edge $u x^{\prime}$. The construction for $\mathbb{D}_{i+1}$ depends on which of these two edges is left of the other at $u$. In Figure 4, we show the first edge of $W[u, y]$ on the left, so the following discussion will be reversed if the edge $u x$ is on the left.

Starting with $u$ and traveling down in the diagram, we always proceed to the right-most lower cover until we reach a minimal element of $P$. In Figure 4 , we suggest that this would result in the chain $(u>v>z>w)$ and it should be clear how the following details should be modified if the actual chain is of a different length.

Starting just above $u$ and headed downward, we insert new points very close to the existing vertices - together with intermediate vertices to ensure that the resulting figure is a diagram. This results in a new minimal element $x^{\prime \prime}$ with $x^{\prime \prime}<$ $x^{\prime} \leq x$ in $P_{i+1}$ and $x^{\prime \prime} \| y$ in $P_{i+1}$. Again, we refer to Figure 4 as an example for how these changes are to be made. Note that no new comparabilities are introduced among the points of $P_{i-1}$ with the addition of these new points.

With this construction in hand, the proof for the reduction is complete.

Given an AFB-diagram for a poset $P$, we know of no simple argument to show that the set of incomparable pairs from $\operatorname{Min}(P) \times P$ can be covered with a bounded number of reversible sets, but in time, we will show that 5 are enough. With the 
reduction, this completes the proof that $\operatorname{dim}(P) \leq 6$ when $P$ is an AFB-poset. However, to simplify the proof, we will first prove a weaker result asserting that the set of incomparable pairs from $\operatorname{Min}(P) \times P$ can be covered by 7 reversible sets. The slight modification necessary to lower 7 to 5 will be presented later.

Lemma 6. Let $\mathbb{D}$ be an $\mathrm{AFB}$-diagram for a poset $P$. Then the set of all incomparable pairs of $P$ in $\operatorname{Min}(P) \times P$ can be covered by 7 reversible sets.

Proof. Clearly, it is enough to prove the lemma when $P$ is connected and has at least two minimal elements. We let $\mathcal{S}_{0}$ denote the set of all incomparable pairs in $\operatorname{Min}(P) \times P$, and we abbreviate the set $\operatorname{Min}(P)$ as $M$.

Since $\mathbb{D}$ is an AFB-diagram, it is easy to see that there is a simple closed curve $\mathcal{E}$ in the plane satisfying the following requirements:

(1) All elements of $M$ are on $\mathcal{E}$.

(2) All elements of $P-M$ are in the interior of $\mathcal{E}$.

(3) If $x$ covered by $y$ in $P$, then all points of the plane which are on the covering edge from $x$ to $y$ in the diagram are in the interior of $\mathcal{E}$, except $x$ when $x \in M$.

We illustrate such a curve in Figure 5 where we show $\mathcal{E}$ using dashed lines. We find it natural to refer to $\mathcal{E}$ as an envelope for $P$.

Starting at an arbitrary minimal element $m_{1}$, we label the elements of $M$ as they appear in a counter-clockwise traversal of $\mathcal{E}$ to obtain a linear order

$$
L=\left[m_{1}<m_{2}<\cdots<m_{n}\right]
$$

on $M$. For each element $y \in P$, we let $M[y]=M \cap D_{P}[y]$.

We will make repeated use of the following elementary proposition. In fact, a stronger result holds, but this is the exact form we need.

Proposition 7. Let $y \in P$, and let $m$ and $m^{\prime}$ be distinct elements of $M$ with $m, m^{\prime} \in D_{P}[y]$. Then let $Z$ be the subposet of $P$ consisting of all elements $z \in P$ with $z \in D_{P}[y]$ such that $m, m^{\prime} \in D_{P}[z]$. Then the subposet $Z$ has a unique minimal element which we will denote $z\left(y, m, m^{\prime}\right)$.

We will also make repeated use of a construction that produces simple closed curves and regions in the plane. Again, let $y \in P$ and let $\left(m, m^{\prime}\right)$ be an ordered pair of distinct elements of $M[y]$. Form a path $\mathcal{E}\left[m, m^{\prime}\right]$ by traversing the simple closed curve $\mathcal{E}$ in a counter-clockwise direction starting at $m$ and stopping at $m^{\prime}$. Now $\mathcal{E}\left[m, m^{\prime}\right]$ and $\mathcal{E}\left[m^{\prime}, m\right]$ share only $m$ and $m^{\prime}$ as endpoints. Their union is the entire curve $\mathcal{E}$.

Let $z=z\left(y, m, m^{\prime}\right)$. We then take witnessing paths from $m$ and $m^{\prime}$ to $z$ using the following convention: If $m$ is left of $m^{\prime}$ in $D_{P}[z]$, then we take $W[m, z]$ as the right-most witnessing path from $m$ to $z$, while we take $W\left[m^{\prime}, z\right]$ as the left-most path from $m^{\prime}$ to $z$. These conventions are reversed if $m^{\prime}$ is left of $m$ in $D_{P}[z]$.

In either situation, the two witnessing paths $W[m, z]$ and $W\left[m^{\prime}, z\right]$ together with the path $\mathcal{E}\left[m, m^{\prime}\right]$ form a simple closed curve which we denote $\mathcal{C}\left(y, m, m^{\prime}\right)$. Also, we let $\mathcal{R}\left(y, m, m^{\prime}\right)$ denote the region in the plane enclosed by $\mathcal{C}\left(y, m, m^{\prime}\right)$. Note that $y$ is on $\mathcal{C}\left(y, m, m^{\prime}\right)$ when $y=z\left(y, m, m^{\prime}\right)$. However, when $y \neq z\left(y, m, m^{\prime}\right), y$ is in the exterior of $\mathcal{R}\left(y, m, m^{\prime}\right)$ when $m$ is left of $m^{\prime}$ in $D_{P}[y]$, and $y$ is in the interior of $\mathcal{R}\left(y, m, m^{\prime}\right)$ when $m$ is right of $m^{\prime}$ in $D_{P}[y]$.

Now back to the argument for covering $\mathcal{S}_{0}$ with 7 reversible sets. We will use the linear order $L$ to label the incomparable pairs in $\mathcal{S}_{0}$ using the following 8 labels: 


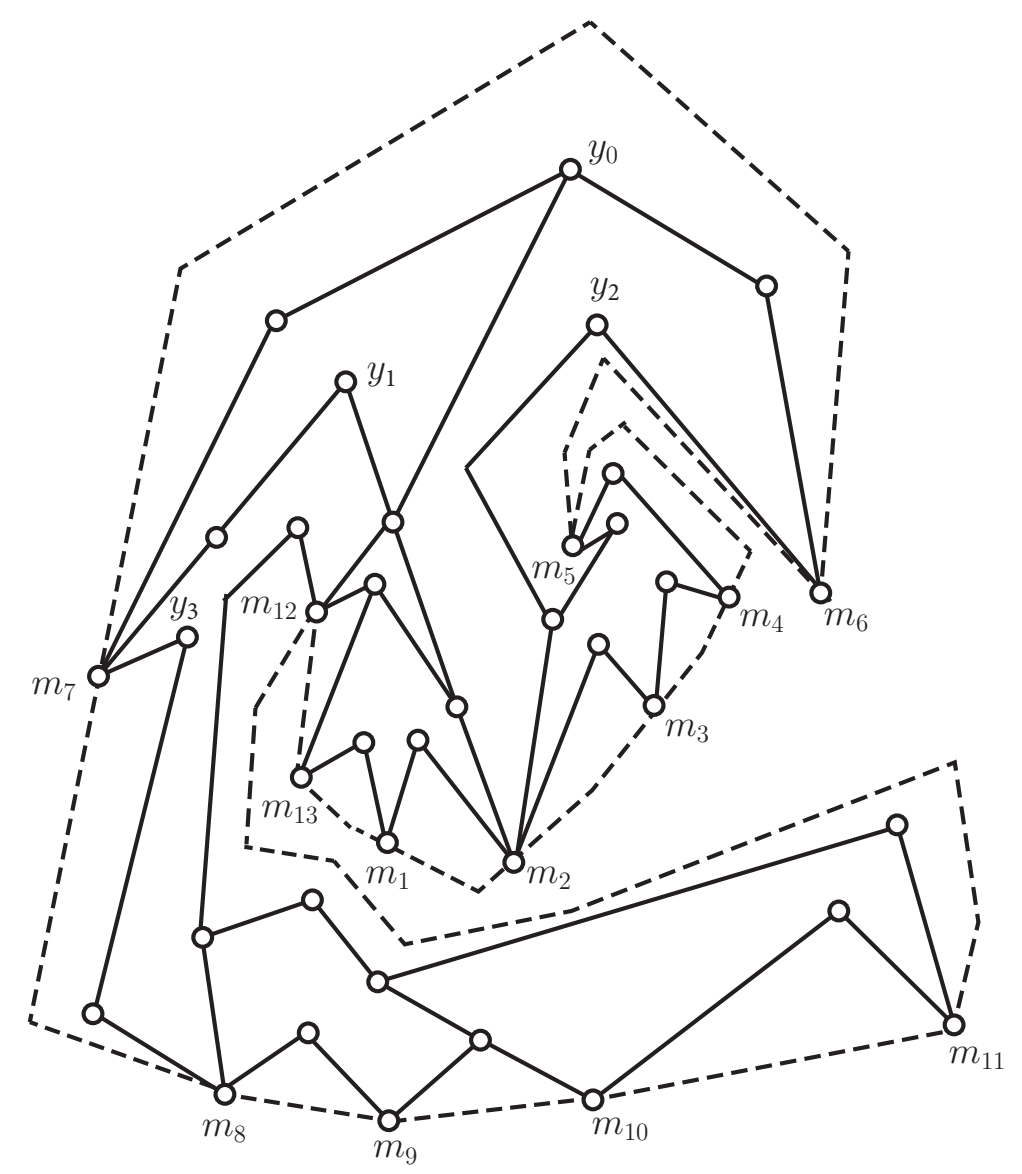

Figure 5. An Envelope for an AFB-poset

\section{$1 A \quad 1 B \quad 1 C \quad 2 A \quad 2 B \quad 2 C \quad 2 D \quad 2 E$.}

The integer part of the label applied to a pair $(x, y)$ depends only on $y$ while the letter in the label depends on both $x$ and $y$.

Let $y$ be an element of $P$. Then the elements of $M[y]$ are linearly ordered from left-to-right in $D_{P}[y]$. We let $s(y)$ and $t(y)$ denote, respectively, the least element and the greatest element of $M[y]$ in this linear order. Let $|M[y]|=r$ and let $\left[u_{1}<u_{2}<\cdots<u_{r}\right]$ be the left-to-right order on $M[y]$ in $D_{P}[y]$, so that $s(y)=u_{1}$ and $t(y)=u_{r}$.

However, the elements of $M[y]$ are also linearly ordered in $L$. Now we let $a(y)$ and $b(y)$ denote, respectively, the least element and the greatest element of $M[y]$ in $L$. Since the envelope $\mathcal{E}$ is traversed in a counter-clockwise manner, it is easy to see that $y$ can be characterized as one of two types, since exactly one of the following two statements holds for $y$ :

Type 1. $u_{1}<u_{2}<\cdots<u_{r}$ in $L$. 
Type 2. There is an integer $j$ with $1<j \leq r$ such that: $u_{j}<u_{j+1}<\cdots<u_{r}<u_{1}<u_{2}<\cdots<u_{j-1}$ in $L$.

We note that an element $y \in P$ is Type 1 when $|M[y]|=1$. In general, when $y$ is Type $1, a(y)=s(y) \leq t(y)=b(y)$ in $L$. When $y$ is Type $2, a(y) \leq t(y)<s(y) \leq$ $b(y)$ in $L$. Also, we observe that either $a(y)=b(y)$ or $a(y)$ is left of $b(y)$ in $D_{P}[y]$ when $y$ is Type 1 . However, $a(y)$ is right of $b(y)$ in $D_{P}[y]$ when $y$ is Type 2.

Now let $(x, y)$ be a pair in $\mathcal{S}_{0}$. If $y$ is Type 1 , we will say that $(x, y)$ is Type $1 A$ if $x<a(y)$ in $L$; Type $1 B$ if $a(y)<x<b(y)$ in $L$; and Type $1 C$ if $x>b(y)$ in $L$. In Figure 5 the elements $y_{2}$ and $y_{3}$ are Type 1 . The pairs $\left(m_{1}, y_{2}\right)$ and $\left(m_{5}, y_{3}\right)$ are Type $1 A$; the pairs $\left(m_{3}, y_{2}\right)$ and $\left(m_{5}, y_{2}\right)$ are Type $1 B$; and the pairs $\left(m_{8}, y_{2}\right)$ and $\left(m_{12}, y_{3}\right)$ are Type $1 C$.

When $y$ is Type 2, we say the pair $(x, y)$ is Type $2 A$ if $x<a(y)$ in $L$; Type $2 B$ if $a(y)<x<t(y)$ in $L$; Type $2 C$ if $t(y)<x<s(y)$ in $L$; Type $2 D$ if $s(y)<x<b(y)$ in $L$; and Type $2 E$ if $x>b(y)$ in $L$. In Figure 5 , the elements $y_{0}$ and $y_{1}$ are Type 2. Now $\left(m_{1}, y_{0}\right)$ and $\left(m_{1}, y_{1}\right)$ are Type $2 A ;\left(m_{4}, y_{0}\right)$ is Type $2 B ;\left(m_{6}, y_{1}\right)$ is Type $2 C$; $\left(m_{8}, y_{0}\right)$ and $\left(m_{10}, y_{1}\right)$ are Type $2 D$; and $\left(m_{13}, y_{0}\right)$ and $\left(m_{13}, y_{1}\right)$ are Type $2 E$.

We then define a covering of $\mathcal{S}_{0}$ by six sets defined as follows:

(1) $\mathcal{S}_{1}$ consists of all Type $1 A$ and $2 A$ pairs.

(2) $\mathcal{S}_{2}$ consists of all Type $1 C$ and $2 E$ pairs.

(3) $\mathcal{S}_{3}$ consists of all Type $1 B$ pairs.

(4) $\mathcal{S}_{4}$ consists of all Type $2 B$ pairs.

(5) $\mathcal{S}_{5}$ consists of all Type $2 D$ pairs.

(6) $\mathcal{S}_{6}$ consists of all Type $2 C$ pairs.

We pause to examine the AFB-poset shown in Figure 6 just to understand that there are obstacles to overcome in covering $\mathcal{S}_{0}$ by a small number of reversible sets. Referring to Figure 6, the set $\mathcal{S}_{1} \cup \mathcal{S}_{2}$ need not be reversible since $\left(x_{4}, y_{4}\right)$ is Type $1 A$ and $\left(x_{5}, y_{5}\right)$ is Type $1 C$, but together these form a strict alternating cycle. Also, $\left(x_{1}, y_{1}\right)$ and $\left(x_{2}, y_{2}\right)$ are Type $2 C$ while $\left(x_{3}, y_{3}\right)$ is Type $2 B$. No reversible set can contain any two of these three pairs so $S_{4} \cup S_{6}$ is not reversible and neither is $S_{6}$.

Despite these challenges, the proof of Lemma 6 and the proof of the (weak) upper bound $\operatorname{dim}(P) \leq 8$ will be complete once we have verified the following claim.

Claim 2. Each of the sets in the family $\left\{\mathcal{S}_{1}, \mathcal{S}_{2}, \mathcal{S}_{3}, \mathcal{S}_{4}, \mathcal{S}_{5}\right\}$ is reversible. Furthermore, the set $\mathcal{S}_{6}$ can be covered by two reversible sets.

Proof. We will examine one set at a time, grouping sets with symmetric arguments.

Case $\mathcal{S}_{1}\left(\mathcal{S}_{2}\right)$. We will first give a proof by contradiction to show that $\mathcal{S}_{1}$ is reversible. The argument for $\mathcal{S}_{2}$ is symmetric. Suppose to the contrary that $\mathcal{S}_{1}$ is not reversible. Let $S=\left\{\left(x_{i}, y_{i}\right): 1 \leq i \leq k\right\}$ be a strict alternating cycle contained in $\mathcal{S}_{1}$. For each $i \in[k]$, let $a_{i}=a\left(y_{i}\right)$, the least element of $M\left[y_{i}\right]$ in the linear order $L$.

For each $i \in[k]$, since $\left(x_{i}, y_{i}\right) \in \mathcal{S}_{1}$, we know that $x_{i}<a_{i}$ in $L$. On the other hand, we know that $x_{i} \leq y_{i+1}$ in $P$. Therefore $a_{i+1} \leq x_{i}$ in $L$. In turn, this implies $a_{i+1}<a_{i}$ in $L$. Clearly, this statement cannot hold for all $i \in[k]$. The contradiction completes the proof for this part of the claim.

Case $\mathcal{S}_{3}$. Now we give a proof by contradiction to show that the set $\mathcal{S}_{3}$ of Type $1 B$ pairs is reversible. This argument will be more substantive than the preceding case. Suppose that $S=\left\{\left(x_{i}, y_{i}\right): 1 \leq i \leq k\right\}$ is a strict alternating cycle of pairs from 


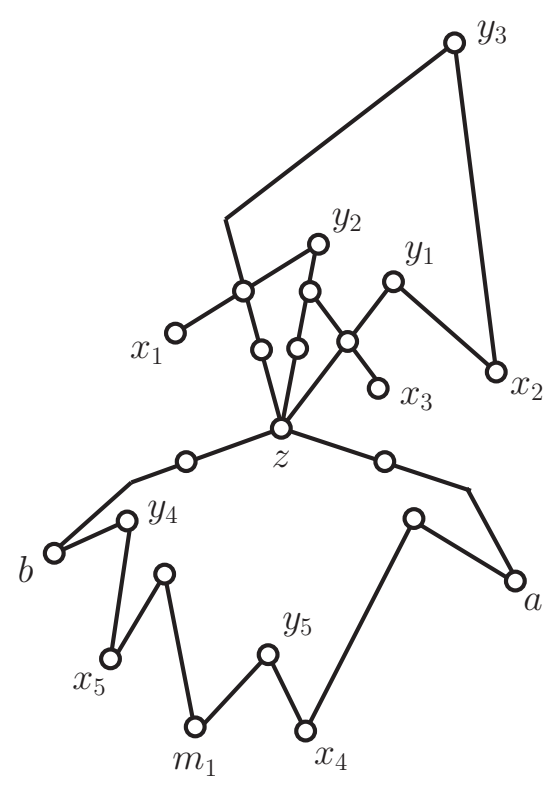

Figure 6. Challenges in Reversing Pairs in $\mathcal{S}_{0}$

$\mathcal{S}_{3}$. For each $i \in[k]$, we let $a_{i}=a\left(y_{i}\right), b_{i}=b\left(y_{i}\right), s_{i}=s\left(y_{i}\right)$ and $t_{i}=t\left(y_{i}\right)$. Since $\left(x_{i}, y_{i}\right) \in \mathcal{S}_{3}$, we know

$$
a_{i}=s_{i}<x_{i}<t_{i}=b_{i} \quad \text { in } L .
$$

Furthermore, we know $a_{i}$ is left of $b_{i}$ in $D_{P}\left[y_{i}\right]$. Let $z_{i}=z\left(y_{i}, a_{i}, b_{i}\right), \mathcal{E}_{i}=\mathcal{E}\left[a_{i}, b_{i}\right]$, $\mathcal{C}_{i}=\mathcal{C}\left(y_{i}, a_{i}, b_{i}\right)$ and $\mathcal{R}_{i}=\mathcal{R}\left(y_{i}, a_{i}, b_{i}\right)$

Now let $i \in[k]$ be arbitrary. Since $a_{i}<x_{i}<b_{i}$ in $L$, we know $x_{i}$ is on the path $\mathcal{E}_{i}$. Since $S$ is a strict alternating cycle, we know $x_{i}<y_{i+1}$ in $P$ and $y_{i} \| y_{i+1}$ in $P$. Let $W\left[x_{i}, y_{i+1}\right]$ be an arbitrary witnessing path. Clearly, $y_{i+1}$ is not a minimal element in $P$, so all points in the plane on the witnessing path $W\left[x_{i}, y_{i+1}\right]$ except $x_{i}$ are in the interior of $\mathcal{C}_{i}$.

Next we consider the set $M\left[y_{i+1}\right]$ which includes $x_{i}$. We assert that all elements of $W\left[y_{i+1}\right]$ are on $\mathcal{E}_{i}$. To see this, suppose $u \in M\left[y_{i+1}\right]$, and $u$ is not on $\mathcal{E}_{i}$. Then $u$ is in the exterior of $\mathcal{C}_{i}$. Let $W\left[u, y_{i+1}\right]$ be an arbitrary witnessing path. Then this path must intersect the boundary of $\mathcal{C}_{i}$, and this forces $u<y_{i}$ in $P$, which is false. The contradiction confirms our assertion.

We conclude that:

$$
a_{i} \leq a_{i+1} \quad \text { and } \quad b_{i+1} \leq b_{i} \quad \text { in } L .
$$

Of course, we also know that $a_{i+1} \leq b_{i}$ in $L$, but we elect to write the two inequalities in (1) in a weak form. Since $i \in[k]$ was arbitrary, these inequalities hold for all $i \in[k]$. We conclude that are minimal elements $a_{0}$ and $b_{0}$ so that $a_{i}=a_{0}$ and $b_{i}=b_{0}$ for each $i \in[k]$. The rules for determining $z_{i}$ and the witnessing paths $W\left[a_{0}, z_{i}\right]$ and $W\left[b_{0}, z\right]$ force $\mathcal{R}_{i+1}$ to be a proper subset of $\mathcal{R}_{i}$. Clearly, this is a 
contradiction since the strict set inclusion statement cannot hold for all $i \in[k]$. This completes the proof that the set $\mathcal{S}_{3}$ consisting of all Type $1 B$ pairs is reversible.

Case $\mathcal{S}_{4}\left(\mathcal{S}_{5}\right)$. Next, we argue by contradiction that the set $\mathcal{S}_{4}$ of all Type $2 B$ pairs is reversible. The argument for the set $\mathcal{S}_{5}$ of all Type $2 D$ pairs is symmetric. Suppose to the contrary that $\mathcal{S}_{4}$ is not reversible, and let $S=\left\{\left(x_{i}, y_{i}\right): 1 \leq i \leq k\right\}$ be a strict alternating cycle contained in $\mathcal{S}_{4}$. We use the same abbreviations as in the preceding case for $a_{i}, b_{i}, s_{i}$ and $t_{i}$. Since $\left(x_{i}, y_{i}\right)$ is Type $2 B$, we know $a_{i}<x_{i}<t_{i}<s_{i} \leq b_{i}$ in $L$.

For each $i \in[k]$, we set $z_{i}=z\left(y_{i}, a_{i}, t_{i}\right), \mathcal{E}_{i}=\mathcal{E}\left[a_{i}, t_{i}\right], \mathcal{C}_{i}=\mathcal{C}\left(y_{i}, a_{i}, t_{i}\right)$ and $\mathcal{R}_{i}=\mathcal{R}\left(y_{i}, a_{i}, t_{i}\right)$. It follows that $y_{i+1}$ is in the interior of $\mathcal{R}_{i}$. We now assert that all points of $M\left[y_{i+1}\right]$ come from $\mathcal{E}\left[a_{i}, b_{i}\right]$. To see this, let $u$ be an element of $M\left[y_{i+1}\right]$ which does not belong to $\mathcal{E}\left(a_{i}, b_{i}\right)$. Then $u$ is in the exterior of $\mathcal{R}_{i}$, and a witnessing path $W\left[u, y_{i+1}\right]$ would have to intersect $\mathcal{C}_{i}$. This forces $u<y_{i}$ in $P$ so that $u \in \mathcal{E}\left(a_{i}, b_{i}\right)$, as desired. In turn, this implies that inequality (1) holds. Since this inequality holds for all $i \in[k]$, we know there are elements $a_{0}, b_{0} \in M$ so that $a_{i}=a_{0}$ and $b_{i}=b_{0}$ for all $i \in[k]$.

Now we assert that $t_{i+1} \leq t_{i}$ for all $i \in[k]$. To the contrary, suppose that $t_{i+1}$ does not belong to $\mathcal{E}\left[a_{0}, t_{i}\right]$. Let $W\left[t_{i+1}, y_{i+1}\right]$ be any witnessing path. Then this path intersects $\mathcal{C}_{i}$. Let $v$ be the unique element of $P$ which is lowest in the plane, and is common to $W\left[t_{i+1}, y_{i+1}\right]$ and the boundary of $\mathcal{C}_{i}$. Clearly, $v<z_{i}$ in $P$. If $v$ is on $W\left[a_{0}, z_{i}\right]$, we conclude that $a_{0}<t_{i+1}<t_{i}$ in the left-to-right order in $D_{P}\left[y_{i}\right]$. This would imply that $t_{i+1}<t_{i}$ in $L$ which is false. We are left to conclude that $v \in W\left[t_{i}, z_{i}\right]$ so that $a_{0}<t_{i}<t_{i+1}$ in $D_{P}\left[y_{i}\right]$, which contradicts the definition of $t_{i}$. We conclude that our assertion that $t_{i+1} \leq t_{i}$ in $L$ is correct. Since $S$ is a strict alternating cycle, we know that there is a point $t_{0} \in M$ so that $t_{i}=t_{0}$ for all $i \in[k]$.

Now the same argument used in proving that the set $\mathcal{S}_{3}$ of all Type $1 B$ pairs is reversible shows that region $\mathcal{R}_{i+1}$ is a proper subset of $\mathcal{R}_{i}$. Clearly, this statement cannot hold for all $i \in[k]$, and this completes the proof that the set $\mathcal{S}_{4}$ consisting of all Type $2 B$ pairs is reversible.

Case $\mathcal{S}_{6}$. Now we turn to the last statement of Claim 2 where we must prove that the set $\mathcal{S}_{6}$ of all Type $2 C$ pairs can be covered by two reversible sets. Note that two Type $2 C$ pairs in Figure 6 shows that $\mathcal{S}_{6}$ may not be reversible.

Let $(x, y)$ be a Type $2 C$ pair, and let $a=a(y), b=b(y)$ and $z=z(y, a, b)$. We will say that $(x, y)$ is left-biased if there is a Type 2 element $y^{\prime} \in P$ such that $\left(1^{\prime}\right) a\left(y^{\prime}\right)=a$ and $b\left(y^{\prime}\right)=b ;\left(2^{\prime}\right) z\left(y^{\prime}, a, b\right)=z ;$ and $\left(3^{\prime}\right) x$ is left of $b(y)$ in $D_{P}\left[y^{\prime}\right]$. Similarly, we will say that $(x, y)$ is right-biased if there is an a Type 2 element $y^{\prime \prime}$ satisfying $\left(1^{\prime \prime}\right) a\left(y^{\prime \prime}\right)=a$ and $b\left(y^{\prime \prime}\right)=b ;\left(2^{\prime \prime}\right) z\left(y^{\prime \prime}, a, b\right)=z$; and also $\left(3^{\prime \prime}\right) x$ is right of $a$ in $D_{P}\left[y^{\prime \prime}\right]$.

We assert that there is no Type $2 C$ pair $(x, y)$ which is both left-biased and right-biased. If this were to happen, we observe that $a, b, z$ belong to both $D_{P}\left[y^{\prime}\right]$ and $D_{P}\left[y^{\prime \prime}\right]$. We would require that $x<a<b$ in the left-to-right order on $D_{P}\left[y^{\prime}\right]$ and $a<b<x$ in the left-to-right order in $D_{P}\left[y^{\prime \prime}\right]$. In particular, both the pairs $(a, x)$ and $(b, x)$ violate Proposition 5 . This proves that the assertion is correct.

We now show that the set $\mathcal{S}^{\prime}$ of all Type $2 C$ pairs which are not right-biased is reversible. The argument to show that the set $\mathcal{S}^{\prime \prime}$ of all Type $2 C$ pairs which are not left-biased is symmetric. Once this has been accomplished, the proof that the set $\mathcal{S}_{6}$ consisting of all Type $2 C$ pairs can be covered by two reversible sets will be complete. 
We argue by contradiction and let $S=\left\{\left(x_{i}, y_{i}\right): 1 \leq i \leq k\right\}$ be a strict alternating cycle of Type $2 C$ pairs, none of which are left-biased. For each $i \in[k]$, we use the now standard abbreviations $a_{i}, b_{i}, s_{i}, t_{i}$. We then take $z_{i}=z\left(y_{i}, a_{i}, b_{i}\right)$, $\mathcal{E}_{i}=\mathcal{E}\left[a_{i}, b_{i}\right], \mathcal{C}_{i}=\mathcal{C}\left(y_{i}, a_{i}, b_{i}\right)$ and $\mathcal{R}_{i}=\mathcal{R}\left(y_{i}, a_{i}, b_{i}\right)$.

Arguments just like those applied earlier show that inequality (1) holds. Therefore, there are elements $a_{0}, b_{0} \in M$ such that $a_{i}=b_{0}$ and $b_{i}=b_{0}$ for all $i \in[k]$.

Now let $i \in[k]$ be arbitrary. We then observe that $\mathcal{R}_{i+1}$ is a proper subset of $\mathcal{R}_{i}$ unless $z_{i+1}=z_{i}$. In this case, $\mathcal{R}_{i+1}=\mathcal{R}_{i}$. It follows that there is an element $z_{0} \in P$ and a simple closed curve $\mathcal{C}_{0}$ enclosing a region $\mathcal{R}_{0}$ so that $z_{i}=z_{0}, \mathcal{C}_{i}=\mathcal{C}_{0}$ and $\mathcal{R}_{i}=\mathcal{R}_{0}$ for all $i \in[k]$.

After a relabeling if necessary, we may assume that $s_{1} \leq s_{i}$ in $L$ for each $i \in[k]$. Then $t_{1}<x_{1}<s_{1}$ in $L$. Since $x_{1}<y_{2}$ in $P$, either $s_{2}<x_{1}<b_{0}$ in $L$ or $a_{0}<x_{1}<t_{2}$ in $L$. If $s_{2} \leq x_{1}<b_{0}$ in $L$, then $s_{2}<s_{1}$ in $L$ which is false. We conclude that $a_{0}<x_{1} \leq t_{2}$ in $L$. Therefore, $x_{1}$ is right of $a_{0}$ in $D_{P}\left[y_{2}\right]$. This shows that $\left(x_{1}, y_{1}\right)$ is right-biased. The contradiction completes the proof.

As promised, we now show how to improve Claim 2 by showing the set $\mathcal{S}_{0}$ can be covered by 5 reversible sets. This will be accomplished by proving the following two claims.

Claim 3. The set $\mathcal{S}_{3} \cup \mathcal{S}_{4}$ of all pairs which are either Type $2 B$ or Type $2 D$ is reversible.

Claim 4. The set $\mathcal{S}_{7}$ consisting of all pairs which are either Type $1 B$ or Type $2 C$ but not right-biased is reversible.

Proof. We first show by contradiction that $\mathcal{S}_{3} \cup \mathcal{S}_{4}$ is reversible. Let $S=\left\{\left(x_{i}, y_{i}\right)\right.$ : $1 \leq i \leq k\}$ be a strict alternating cycle of pairs from $\mathcal{S}_{3} \cup \mathcal{S}_{4}$ In view of our earlier arguments, there must be at least one pair in $S$ of Type $2 B$ and at least one pair of Type $2 D$.

The abbreviations $a_{i}, b_{i}, s_{i}, t_{i}$ are just as before. Now we know that $a_{i} \leq t_{i}<$ $s_{i} \leq b_{i}$ in $L$. Furthermore, if $\left(x_{i}, y_{i}\right)$ is Type $2 B$, we know $a_{i}<x_{i}<t_{i}$ in $L$, and if $\left(x_{i}, y_{i}\right)$ is Type $2 D$, we know $s_{i}<x_{i}<b_{i}$ in $L$.

Now let $i \in[k]$. If $a_{i}=t_{i}$, we set $z_{i}=a_{i}$, and we let $\mathcal{R}_{i}$ be the region in the plane consisting only of the point $a_{i}$. If $a_{i}<t_{i}$ in $L$, we set $z_{i}=z\left(y_{i}, a_{i}, t_{i}\right)$, $\mathcal{C}_{i}=\mathcal{C}\left(y_{i}, a_{i}, b_{i}\right)$ and $\mathcal{R}_{i}=\mathcal{R}\left(y_{i}, a_{i}, t_{i}\right)$. Analogously, if $s_{i}=t_{i}$, we set $v_{i}=b_{i}$ and we take $\mathcal{T}_{i}$ as the region in the plane consisting only of the point $b_{i}$. If $s_{i}<b_{i}$ in $L$, we let $v_{i}=z\left(y_{i}, s_{i}, b_{i}\right), \mathcal{D}_{i}=\mathcal{C}\left(y_{i}, s_{i}, b_{i}\right)$ and $\mathcal{T}_{i}=\mathcal{R}\left(y_{i}, s_{i}, t_{i}\right)$,

Repeating arguments already presented, we quickly learn that there are elements $a_{0}, b_{0}, s_{0}, t_{0} \in M$, elements $z_{0}, v_{0} \in P$, simple closed curves $\mathcal{C}_{0}$ and $\mathcal{D}_{0}$ and regions $\mathcal{R}_{0}, \mathcal{T}_{0}$ so that $a_{i}=a_{0}, b_{i}=b_{0}, z_{i}=z_{0}, v_{i}=v_{0}, \mathcal{C}_{i}=\mathcal{C}_{0}, \mathcal{R}_{i}=\mathcal{R}_{0}, \mathcal{D}_{i}=\mathcal{D}_{0}$, and $\mathcal{T}_{i}=\mathcal{T}_{0}$ for all $i \in[k]$.

If $i \in[k]$ and $\left(x_{i}, y_{i}\right)$ is Type $2 B$, then it is easy to see that $\mathcal{R}_{i+1} \subsetneq \mathcal{R}_{i}$ while $\mathcal{T}_{i+1}=\mathcal{T}_{i}$. Analogously, if $\left(x_{i}, y_{i}\right)$ is Type $2 D$ then $\mathcal{T}_{i+1} \subsetneq \mathcal{T}_{i}$ while $\mathcal{R}_{i+1}=\mathcal{R}_{i}$. Clearly, these statements result in a contradiction, so we have completed the proof that $\mathcal{S}_{3} \cup \mathcal{S}_{4}$ is reversible.

Now we prove by contradiction that $\mathcal{S}_{7}$, which consists of all Type $1 B$ pairs and all Type $2 C$ pairs which are not right-biased is reversible. Let $S=\left\{\left(x_{i}, y_{i}\right): 1 \leq\right.$ $i \leq k\}$ be a strict alternating cycle contained in $\mathcal{S}_{7}$. Then we know that $S$ contains both a Type $1 B$ pair and a Type $2 C$ pair. 
Now suppose that $i \in[k]$. We set $a_{i}=a\left(y_{i}\right), b_{i}=b\left(y_{i}\right), z_{i}=z\left(y_{i}, a_{i}, b_{i}\right)$, $\mathcal{C}_{i}=\mathcal{C}\left(y_{i}, z_{i}, b_{i}\right)$ and $\mathcal{R}_{i}=\mathcal{R}\left(y_{i}, a_{i}, b_{i}\right)$. Then $y_{i+1}$ is in the interior of $\mathcal{R}_{i}$ and all elements of $M\left[y_{i+1}\right]$ are on the path $\mathcal{E}\left(a_{i}, b_{i}\right)$. It follows that the inequalities in (1) hold. We conclude that there are elements $a_{0}, b_{0} \in M$ such that $a_{i}=a_{0}$ and $b_{i}=b_{0}$ for all $i \in[k]$.

Let $i$ and $j$ be integers in $[k]$ so that $\left(x_{i}, y_{i}\right)$ is Type $1 B$ and $\left(x_{j}, y_{j}\right)$ is Type $2 C$. Then $a_{0}$ is left of $b_{0}$ in $D_{P}\left[y_{i}\right]$ and $a_{0}$ is right of $b_{0}$ in $D_{P}\left[y_{j}\right]$. These statements contradict Proposition 5. With these observations, the proof of Claim 3 is complete. This also completes the proof of our main theorem.

\section{Closing Comments and Open Problems}

We pause to explain our motivation in studying the class of AFB-posets. Let $P$ be a planar poset and let $x_{0}$ be an arbitrary minimal element of $P$. Then set $A_{0}=\left\{x_{0}\right\}$ and let $B_{0}$ consist of all elements $y$ in $P$ such that $y>x_{0}$ in $P$. If $i \geq 0$ and we have defined a sequence $\left(A_{0}, B_{0}, A_{1}, B_{1}, \ldots, A_{i}, B_{i}\right)$ of pairwise disjoint subposets of $P$ and their union is a proper connected subposet $Q$ of $P$, we let $A_{i+1}$ consist of all elements $x \in P-Q$ for which there is some $y \in B_{i}$ such that $x<y$ in $P$. Also, when $Q \cup A_{i+1}$ is a proper subposet of $P$, we take $B_{i+1}$ as the set of all $y \in P-\left(Q \cup A_{i+1}\right)$ for which there is some $x \in A_{i+1}$ for which $x<y$ in $P$.

The resulting partition of $P$ is now known as an unfolding of $P$, and this concept has been used in several papers, including [14, [12] and [7]. The key feature for our purposes is that for all $i \geq 0$, the subposet $B_{i}$ is an AFB-poset, and the dual of the subposet $A_{i}$ is an AFB-poset. As is well known there is some $i \geq 0$ for which:

$$
\max \left\{\operatorname{dim}\left(A_{i} \cup B_{i}\right), \operatorname{dim}\left(B_{i} \cup A_{i+1}\right)\right\} \geq \operatorname{dim}(P) / 2 .
$$

It follows that when the dimension of $P$ is very large, we now know that there is a subposet of $P$ in which the difficulty of the dimension problem has a "bipartite flavor," i.e., the poset is the union of two relatively simple subposets, one a down set and the other an up set and the challenge is to reverse incomparable pairs of the form $(x, y)$ where $x$ is in the down set and $y$ is in the up set. Reversing the remaining incomparable pairs takes at most 6 linear extensions.

Our motivation for this line of research has from the outset been to develop machinery for attacking the following long-standing and apparently quite challenging conjectures:

Conjecture 8. A planar poset with large dimension contains a large standard example, i.e., for every $d \geq 2$, there exists a constant $d_{0}$ so that if $P$ is a planar poset and $\operatorname{dim}(P) \geq d_{0}$, then $P$ contains the standard example $S_{d}$ as a subposet.

We believe, but cannot be certain, that the first reference to this conjecture is on page 119 in [15], as it has become part of the folklore of the subject.

In fact, probably the following considerably stronger conjecture is true.

Conjecture 9. For every pair $(n, d)$ of positive integers with $d \geq 2$, there is an integer $d_{0}$ so that if $P$ is a poset and $\operatorname{dim}(P) \geq d_{0}$, then either $P$ contains the standard example $S_{d}$ or the cover graph of $P$ contains a $K_{n}$ minor. 
In just the last two years, there has been considerable interest in two variants of the original Dushnik-Miller notion of dimension. They are called Boolean dimension and local dimension. We refer readers to [17, 4, 11] and [2] for definitions and results.

Specific to our interests here is the proof by Bosek, Grytczuk and Trotter 2 that local dimension is not bounded for planar posets. The following conjecture is due to Nešetřil and Pudlak and is given in question form in their 1989 paper [13] in which the concept of Boolean dimension is first introduced.

Conjecture 10. The Boolean dimension of planar posets is bounded, i.e., there is a constant $d_{0}$ so that if $P$ is a planar poset, then the Boolean dimension of $P$ is at most $d_{0}$.

We believe that the results presented here will prove useful in attacking this conjecture with the assistance of the concept of unfolding.

\section{REFERENCES}

[1] F. Barrera-Cruz, T. Prag, H. C. Smith and W. T. Trotter. Comparing Dushnik-Miller dimension, Boolean dimension and local dimension, submitted (available on the arXiv at 1710.09467).

[2] B. Bosek, J. Grytczuk and W. T. Trotter, Local dimension is unbounded for planar posets, submitted (available on the arXiv at 1712.06099).

[3] B. Dushnik and E. W. Miller, Partially ordered sets, Amer. J. Math. 63 (1941), 600-610.

[4] S. Felsner, T. Mészáros and P. Micek, Boolean dimension and tree-width, submitted (available on the arXiv at 1707.06114).

[5] S. Felsner, W. T. Trotter and V. Wiechert, The dimension of posets with planar cover graphs, Graphs and Combin. 31 (2015), 927-939.

[6] G. Joret, P. Micek, K. G. Milans, W. T. Trotter, B. Walczak and R. Wang, Tree-width and dimension, Combinatorica 36 (2016), 431-450.

[7] G. Joret, P. Micek and V. Wiechert, Planar posets have dimension at most linear in their height, SIAM J. Discrete Math. 31 (2017), 2754-2790.

[8] G. Joret, P. Micek and V. Wiechert, Sparsity and dimension, Combinatorica, to appear (available on the arXiv at 1507.01120).

[9] G. Joret, P. Micek, W. T. Trotter, R. Wang and V. Wiechert, On the dimension of posets with cover graphs of tree-width 2, Order 34 (2017), 185-234.

[10] D. Kelly, On the dimension of partially ordered sets, Discrete Math. 35 (1981), 135-156.

[11] T. Mészáros, P. Micek and W. T. Trotter, Boolean dimension, components and blocks, submitted (available on the arXiv at 1801.00288).

[12] P. Micek and V. Wiechert, Topological minors of cover graphs and dimension, J. Graph Theory 86 (2017), 415-420.

[13] A Note on Boolean dimension of posets, in Irregularities of Partitions, Vol. 8 of Algorithms and Combinatorics, G. Halász and V. T. Sós, eds., Springer, Berlin (1989), 137-140.

[14] N. Streib and W. T. Trotter, Dimension and height for posets with planar cover graphs, European J. Combin. 3 (2014), 474-489.

[15] W. T. Trotter, Combinatorics and Partially Ordered Sets: Dimension Theory, The Johns Hopkins University Press, Baltimore, 1992.

[16] W. T. Trotter and J. I. Moore, The dimension of planar posets, J. Combin. Theory Ser. B 21 (1977), 51-67.

[17] W. T. Trotter and B. Walczak, Boolean dimension and local dimension, Extended abstract published at Electronic Notes in Discrete Mathematics 61 (2017), 1047-1053 (with B. Walczak). Full journal version is under review and is available on the arXiv at 1705.09167.

[18] W. T. Trotter and R. Wang, Planar posets, dimension, breadth and the number of minimal elements, Order 33 (2016), 333-346.

[19] W. T. Trotter, B. Walczak and R. Wang, Dimension and cut vertices: An application of Ramsey theory, in Connections in Discrete Mathematics, S. Butler, et al., eds., Cambridge University Press, to appear. 
[20] B. Walczak, Minors and dimension, J. Combin. Theory Ser. B 122 (2017) 668-689.

(Biro) Department of Mathematics, University of Louisville, Louisville, Kentucky 40292, U.S.A.

E-mail address: csaba.biro@louisville.edu

(Bosek) Theoretical Computer Science Department, Faculty of Mathematics and Computer Science, Jagiellonian University, Kraków, Poland

E-mail address: bosek@tcs.uj.edu.pl

(Smith) Department of Mathematics and Computer Science, Davidson College, DavidSOn, North CARolina 28035, U.S.A.

E-mail address: hcsmith@davidson.edu

(Trotter) School of Mathematics, Georgia Institute of Technology, Atlanta, GeorGIA 30332 , U.S.A.

E-mail address: trotter@math.gatech.edu

(Wang) Blizzard Entertainment, Irvine, California, U.S.A.

E-mail address: rwang49@math.gatech.edu

(Young) Pacific Northwest National Laboratory, Richland, Washington 99352, U.S.A.

E-mail address: stephen.young@pnnl.gov 